\title{
EchoGéo
}

\section{Le tournant environnemental en Amazonie : ampleur et limites du découplage entre production et déforestation}

François Laurent, Damien Arvor, Marion Daugeard, Reinis Osis, Isabelle Tritsch, Emilie Coudel, Marie-Gabrielle Piketty, Marc Piraux, Cecilia Viana, Vincent Dubreuil, Ali F. Hasan and François Messner

\section{(2) OpenEdition}

1 Journals

Electronic version

URL: https://journals.openedition.org/echogeo/15035

DOI: $10.4000 /$ echogeo. 15035

ISSN: 1963-1197

Publisher

Pôle de recherche pour l'organisation et la diffusion de l'information géographique (CNRS UMR 8586)

Electronic reference

François Laurent, Damien Arvor, Marion Daugeard, Reinis Osis, Isabelle Tritsch, Emilie Coudel, MarieGabrielle Piketty, Marc Piraux, Cecilia Viana, Vincent Dubreuil, Ali F. Hasan and François Messner, "Le tournant environnemental en Amazonie : ampleur et limites du découplage entre production et déforestation", EchoGéo [Online], 41 | 2017, Online since 28 September 2017, connection on 31 July 2021. URL: http://journals.openedition.org/echogeo/15035 ; DOI: https://doi.org/10.4000/echogeo. 15035

This text was automatically generated on 31 July 2021.

EchoGéo est mis à disposition selon les termes de la licence Creative Commons Attribution - Pas d'Utilisation Commerciale - Pas de Modification 4.0 International (CC BY-NC-ND) 


\title{
Le tournant environnemental en Amazonie : ampleur et limites du découplage entre production et déforestation
}

\author{
François Laurent, Damien Arvor, Marion Daugeard, Reinis Osis, Isabelle \\ Tritsch, Emilie Coudel, Marie-Gabrielle Piketty, Marc Piraux, Cecilia Viana, \\ Vincent Dubreuil, Ali F. Hasan and François Messner
}

Les recherches sur la municipalité de Paragominas ont été financées par l'Agence Nationale de la Recherche, pour le projet ECOTERA (ECOefficiences et développement TERritorial en Amazonie brésilienne; ANR-13-AGRO-0003). Les recherches à l'échelle de l'Amazonie ont été menées dans le cadre des projets ANR DURAMAZ-2 (ANR-11-BSH1-0003) et H2020-MSCA-RISE-2015 ODYSSEA (Référence du projet: 691053).

\section{Introduction}

1 La question de la déforestation en Amazonie cristallise les préoccupations internationales sur le changement climatique et l'érosion de la biodiversité. Depuis la fin des années 1990, le gouvernement fédéral brésilien a accordé une priorité grandissante à la lutte contre la déforestation. A partir de 2008, il a ciblé ses contrôles dans certaines municipalités ${ }^{1}$ sur la base d'indicateurs reflétant l'intensité de la déforestation pour s'assurer du respect des réglementations environnementales (Piketty et al., 2017). En parallèle, des ONG internationales ont exercé une pression sur les filières du soja et de la viande bovine conduisant ces dernières à s'engager à réduire leurs impacts sur la forêt (Gibbs et al., 2015 ; Gibbs et al., 2016). Le déboisement a baissé entre 2005 et 2015 de près de $80 \%$ par rapport à la décennie précédente sans que cela ne freine la croissance économique de la région (Macedo et al., 2012 ; Lapola et al., 2014; Nepstad et al., 2014), le gouvernement cherchant en parallèle à favoriser les activités agricoles et d'élevage, sources de commodities pour le marché mondial. Dans ce 
contexte, la question est alors de savoir quelle est l'ampleur de ce tournant environnemental ? Comment la croissance du secteur agricole associée à une baisse de la déforestation représente - $t$-elle une évolution des formes du développement (c'est-àdire au-delà de la croissance économique, un changement de modes de production et de gouvernance) ? Ce développement concerne-t-il les différents groupes sociaux ? Et quelle est la place du territoire dans cette évolution? Par le terme de «tournant environnemental ", nous désignons ici des changements de rapports entre l'activité agricole et l'environnement liés aux contraintes, pressions et demandes émanant de la réglementation ou des marchés et dans une certaine mesure, que nous n'évaluerons pas ici, d'une prise de conscience chez les producteurs que croissance économique ne rime pas forcément avec déforestation.

2 Les effets des engagements des pouvoirs publics et des filières peuvent être lus à différentes échelles spatiales. La première est régionale et correspond à l'Amazonie Légale ${ }^{2}$. Cette échelle permet d'évaluer les conséquences des engagements sur le lien entre croissance économique et déforestation. La seconde échelle est locale, elle vise à approfondir le rôle des acteurs locaux, leur adaptation aux politiques et leur capacité à se mettre en conformité avec les réglementations environnementales. Elle permet de lire les effets des nouvelles dynamiques de développement agricole sur l'organisation de l'espace. Nous avons choisi pour illustrer ce niveau la municipalité de Paragominas, dans l'État du Pará. Cette municipalité est considérée comme un modèle en matière de baisse de la déforestation et de gouvernance environnementale en Amazonie. Elle montre ainsi des processus d'adaptation aux nouveaux cadres environnementaux qui ailleurs sont plus ténus, voire encore inexistants. Paragominas a donc été retenue dans notre analyse non pas pour sa représentativité mais pour identifier les avancées réalisées et les limites et pour les expliquer dans un espace où ces processus sont plus marqués et donc plus lisibles.

3 Les résultats présentés dans cet article s'appuient sur une analyse des données publiques de l'IBGE en ce qui concerne les statistiques démographiques et agricoles (IBGE, 2010, 2017a 2017b), sur le traitement d'images satellites MODIS ou l'exploitation des données Terraclass (INPE, 2014) et PRODES (INPE, 2016) pour l'occupation du sol et son évolution et sur des entretiens réalisés avec des producteurs et des acteurs territoriaux du développement agricole et de la protection de l'environnement.

4 Fruit de la collaboration de chercheurs impliqués dans divers projets de recherches internationaux (ANR DURAMAZ-2, ANR ECOTERA, H2020 ODYSSEA), l'objectif de cet article est de mutualiser les connaissances acquises autour des enjeux du développement et de la préservation environnementale en Amazonie. Il s'agit ainsi d'analyser les avancées réalisées en matière de préservation de l'environnement au regard du développement du secteur agricole, mais aussi leurs limites notamment liées aux inégalités sociales. Pour ce faire, le plan s'organise autour de trois points : (i) les engagements publics et privés en matière d'environnement et de développement durable, notamment au cours des années Lula, (ii) les résultats de l'évolution en cours visibles aux échelles régionale et territoriale, (iii) les inégalités sociales persistantes et leurs conséquences environnementales. 


\section{L'engagement des pouvoirs publics, des filières et des acteurs territoriaux contre la déforestation marque un tournant en Amazonie}

5 La diminution des taux de déforestation en Amazonie depuis 2005 témoigne d'un tournant environnemental visant à mieux maîtriser l'expansion de la frontière agricole. Il est la conséquence d'un ensemble de mesures prises par divers acteurs à plusieurs échelles, sous l'influence de la société civile (ONG) et de la pression internationale. Il est notamment marqué par une évolution des politiques publiques nationales, par l'engagement des filières du soja et de la viande bovine (Rudorff et al., 2011 ; Arima et al., 2014), mais est aussi accompagné, comme nous le verrons dans le cas de Paragominas, d'une implication des acteurs territoriaux.

\section{Le tournant environnemental en Amazonie}

6 L'occupation de l'Amazonie Légale, planifiée par l'État brésilien, commence dès les années 1930 (illustration 1).

Illustration 1 - Chronologie (non-exhaustive) des politiques publiques et des plans, programmes et instruments (PPI) menés en Amazonie, dans l'État du Pará et à Paragominas

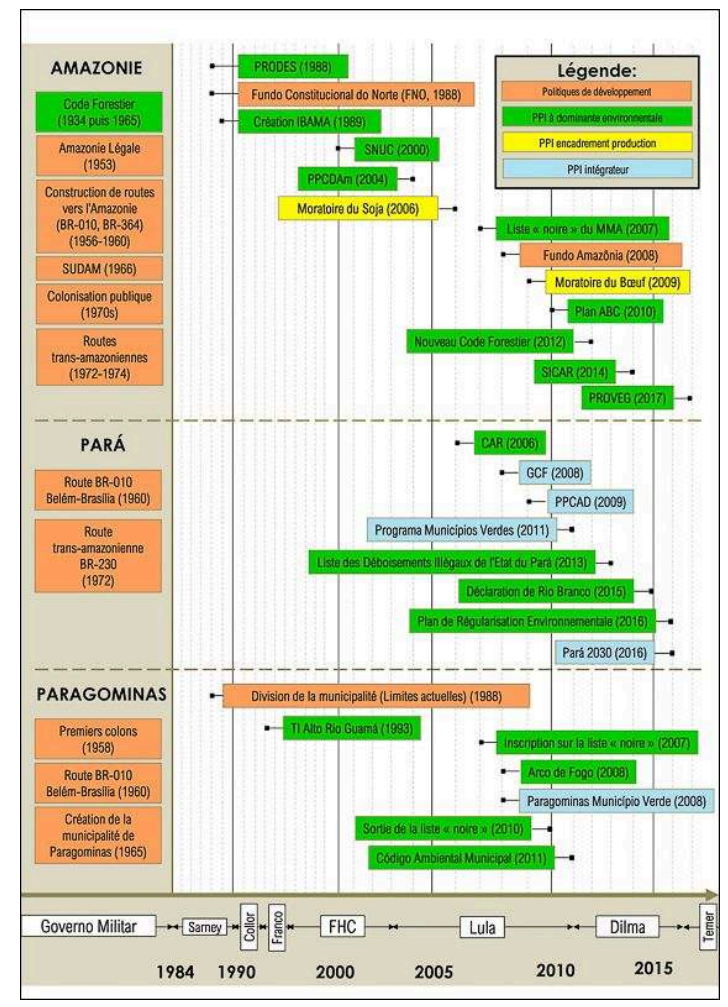

Les politiques de développement sont clairement orientées vers la dimension économique sans faire mention de la dimension environnementale. Les PPI à dominante environnementale visent au contraire à établir les règles de préservation de l'environnement ainsi qu'à contrôler et punir les infractions. Les PPI d'encadrement de la production visent à contraindre le développement agricole en le forçant à respecter les règles environnementales. Enfin les PPI "intégrateurs" prônent un développement intégrant (au moins) les deux dimensions: économiques et environnementales. 
7 Son développement fait l'objet de programmes nationaux pluriannuels tels que le PIN (Plano de Integração Nacional, 1970-1974) ou le PND (Plano Nacional de Desenvolvimento, 1975-1979), qui s'appuient notamment sur l'ouverture de "l'Amazonie des routes » le long desquelles sont implantés de nombreux projets de colonisation publique ou privée (Théry, 1997 ; de Mello et Théry, 2003 ; Dubreuil, 2002 ; Coy et al., 2017). La déforestation est principalement associée à la création de pâturages, l'élevage bovin extensif étant favorisé par le marché de la viande, la sécurisation des revenus et le savoir-faire des migrants, issus généralement de régions d'élevage (Piketty et al., 2005). Dans ce contexte, outre l'intérêt pour la production agricole, les conflits pour l'appropriation du sol incitent également à la déforestation, le nouvel occupant cherchant d'abord à marquer son territoire avant de le valoriser (Tourrand et al., 2013). Les préoccupations environnementales sont alors faibles et les réglementations peu respectées. Les versions successives du Code Forestier (1934, 1965 et les nombreux décrets et mesures provisoires) qui régit la conservation des forêts privées, tout comme la mise en place, à la fin des années 1980 d'un système de suivi de la déforestation par satellite (projet PRODES; INPE, 2016) n'a qu'un impact limité sur l'avancée de la frontière agricole. Cette phase repose donc sur une expansion agro-industrielle (Nepstad et al., 2014) aux dépens de l'environnement : la législation sur la forêt est perçue comme un obstacle au développement par les filières agricoles, tandis que les critiques envers l'agro-industrie prennent de l'ampleur en raison de la déforestation et de l'expulsion des petits producteurs des espaces dont elle prend le contrôle (Lapola et al., 2014).

8 Face à ces pressions, à partir de 2004, le nouveau gouvernement fédéral du président Lula instaure le Plan pour la Protection et le Contrôle de la Déforestation en Amazonie (PPCDAm) et, à partir de 2007, des mesures fortement contraignantes (Décret présidentiel $n^{\circ} 6.321$ ) ciblent une liste "noire" de municipalités amazoniennes sur la base d'indicateurs reflétant l'intensité de la déforestation (36 municipalités étaient concernées à l'époque). Les filières agricoles impliquées sur ces territoires sont également tenues responsables du délit de déforestation illégale. En 2008, au cours de l'opération spéciale Arco de fogo (Arc de feu), des contrôles sur le terrain de l'IBAMA (Institut Brésilien de l'Environnement) et de la Police Fédérale ciblent ces territoires : les producteurs responsables de déforestation illégale sont inculpés et les scieries et fours à charbon végétal illégaux sont fermés (Barreto et Silva, 2010 ; Sist et al., 2013). En renforcement de ces opérations coup de poing, la Banque Centrale (décret $n^{\circ} 3.468$ ) interdit en 2008 l'octroi de crédit agricole sans preuve de titre foncier et de légalité environnementale, documents rares en Amazonie, bloquant notamment les crédits de campagne de nombreux producteurs.

Aux mesures gouvernementales, s'ajoutent celles des filières sous la pression des ONG environnementalistes (Greenpeace International, 2006). Le Moratoire du soja, lancé en 2006, est l'une des initiatives en réponse à ces pressions: les entreprises signataires s'engagent à ne pas acheter de soja provenant de terres déboisées après le 26 juillet $2006^{3}$ (Rudorff et al., 2011). Le Groupe de Travail sur le Soja (GTS) est chargé de veiller au suivi du moratoire dans les municipalités où plus de 5000 hectares sont plantés en soja (Gibbs et al., 2015). Ces mesures ont eu un rôle important (Butler et Laurance, 2008; Rudorff et al., 2011) même si le respect des règles du moratoire ne signifie pas un respect total du Code Forestier (Azevedo et al., 2015, 2017 ; Gibbs et al., 2015; Piketty et al., 2015). 
10 En 2009, c'est au tour du secteur de la viande: le gouvernement fédéral rend les abattoirs co-responsables de crime environnemental s'ils achètent des animaux provenant de déforestations illégales (Sist et al., 2013; Gibbs et al., 2016). Ils doivent signer un TAC (Termo de Ajustamento de Conduta), un engagement à acheter des animaux élevés dans des propriétés enregistrées dans le CAR (Cadastro Ambiental Rural) ${ }^{4}$ et possédant une Licence Environnementale Rurale (LAR) ${ }^{5}$. Rapidement, ces engagements sont reproduits dans les autres États amazoniens. Les plus grands abattoirs brésiliens (Marfrig, Minerva, JBS and Bertin) signent également un accord avec Greenpeace reprenant les exigences du TAC en les étendant aux fournisseurs indirects des abattoirs (les producteurs de veaux), ce qui implique la traçabilité des animaux (Brannstrom et al., 2012 ; Drigo, 2013; Greenpeace International, 2009). Ils s'engagent en outre à respecter la législation environnementale en vigueur et à se mettre en conformité le cas échéant. Le CAR est finalement rendu obligatoire en 2012 pour tous les propriétaires ruraux, lors de la réforme du Code Forestier. Ce dernier, fruit d'un intense processus de négociation politique, devient le principal instrument légal qui régule l'usage des sols dans les exploitations rurales ${ }^{6}$. La mise en place de ce cadastre devient la priorité politique en Amazonie et est en grande partie financée par des projets du Fundo Amazônia ${ }^{7}$. Il constitue en outre un élément déterminant pour accéder au crédit agricole. En février 2017, plus de 126 millions d'hectares étaient enregistrés dans les bases du $\mathrm{CAR}^{8}$ dans la région Nord (Amazonie), la plaçant en tête des régions brésiliennes avec plus d'un tiers des 402 millions d'hectares cadastrés au Brésil9 ${ }^{9}$.

11 Ainsi, après une phase d'expansion agro-industrielle d'une rapidité et d'une ampleur sans précédent, des politiques publiques plus intégrées se combinent avec un certain succès avec des engagements des filières pour enrayer la déforestation et orienter les producteurs vers un développement agricole respectant la législation environnementale (Piketty et al., 2015).

\section{Importance des démarches territoriales : le cas de Paragominas}

12 Paragominas (fondée en 1965, $19342 \mathrm{~km}^{2}, 108547$ habitants en 2016, selon l'IBGE, 2017a) est une municipalité située au nord-est de l'État du Pará (illustration 2). L'économie locale est basée sur l'agriculture, l'élevage, l'extraction de bauxite et les services associés. Les grandes propriétés, orientées vers l'élevage bovin extensif (342 000 têtes de bovins recensées en 2015 ; IBGE, 2017a) et la production de soja et de maïs (respectivement 275500 et 121000 tonnes en 2015 ; IBGE, 2017b) dominent l'espace agricole, notamment à proximité des routes principales. Les zones de forêt les mieux préservées se situent à l'extrême nord-est de la municipalité, du fait d'une terre indigène (Terra Indígena Alto Rio Gúama) et au sud-ouest dans une zone de 140658 ha exploitée par une grande entreprise de bois, certifiée FSC depuis 2001 (Pinto et al., 2009). Sur la carte (illustration 2), la forêt recouvre différents types : forêt primaire, forêt secondaire et plantations sylvicoles commerciales. L'espace agricole est très largement dominé par la grande propriété, l'agriculture familiale n'occupant que $5 \%$ des surfaces de la municipalité (illustration 3). À Paragominas, une propriété est considérée en agriculture familiale si sa surface est inférieure à 220 ha (le módulo fiscal étant de $55 \mathrm{ha}$ ); il s'agit bien sûr d'un seuil théorique masquant une diversité de modes de production. Les périmètres de réforme agraire, où sont localisés la grande majorité des petits producteurs, sont situés sur les marges de la municipalité, distants de la ville (illustration 2). Outre les cultures de subsistance (notamment manioc), ceux-ci y 
pratiquent l'élevage bovin (viande et lait), la culture du poivre et la cueillette de l'açail ${ }^{10}$ le long des cours d'eau.

Illustration 2 - Occupation du sol à Paragominas en 2015 et en Amazonie légale en 2014

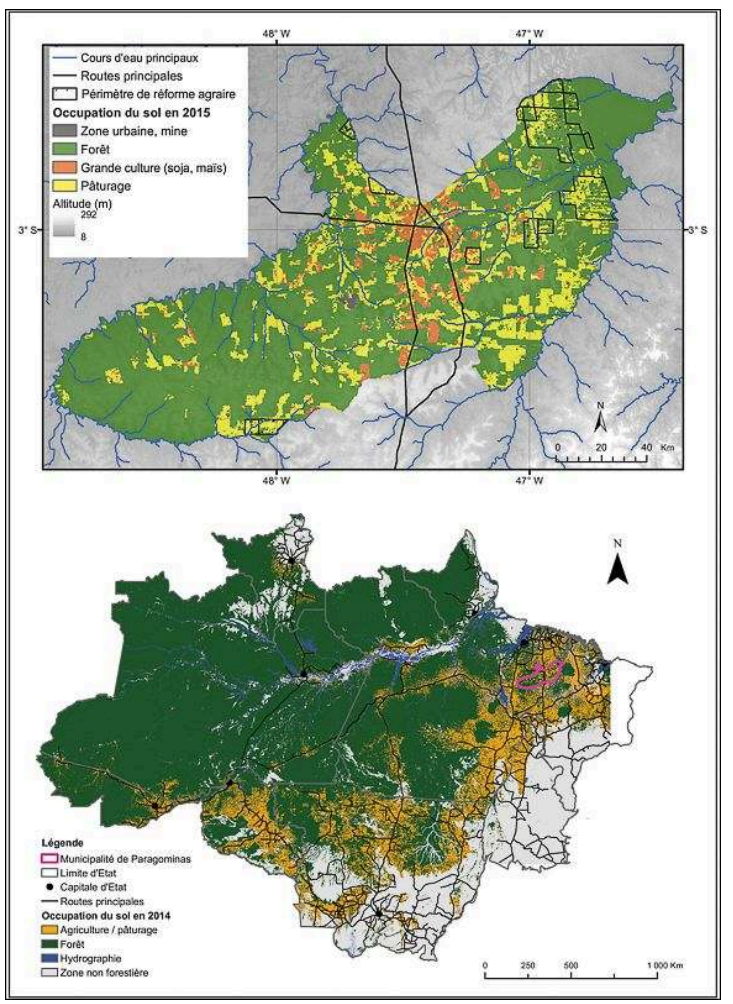

Sources de données pour Paragominas : occupation du sol issue de traitements d'images MODIS (classification réalisée par R. Osis) et altitude issue de SRTM 90 m (Jarvis et al., 2008) - pour l'Amazonie Légale : INPE (2014). 
Illustration 3 - Surface relative de chaque classe de taille de propriété (en ha) à Paragominas

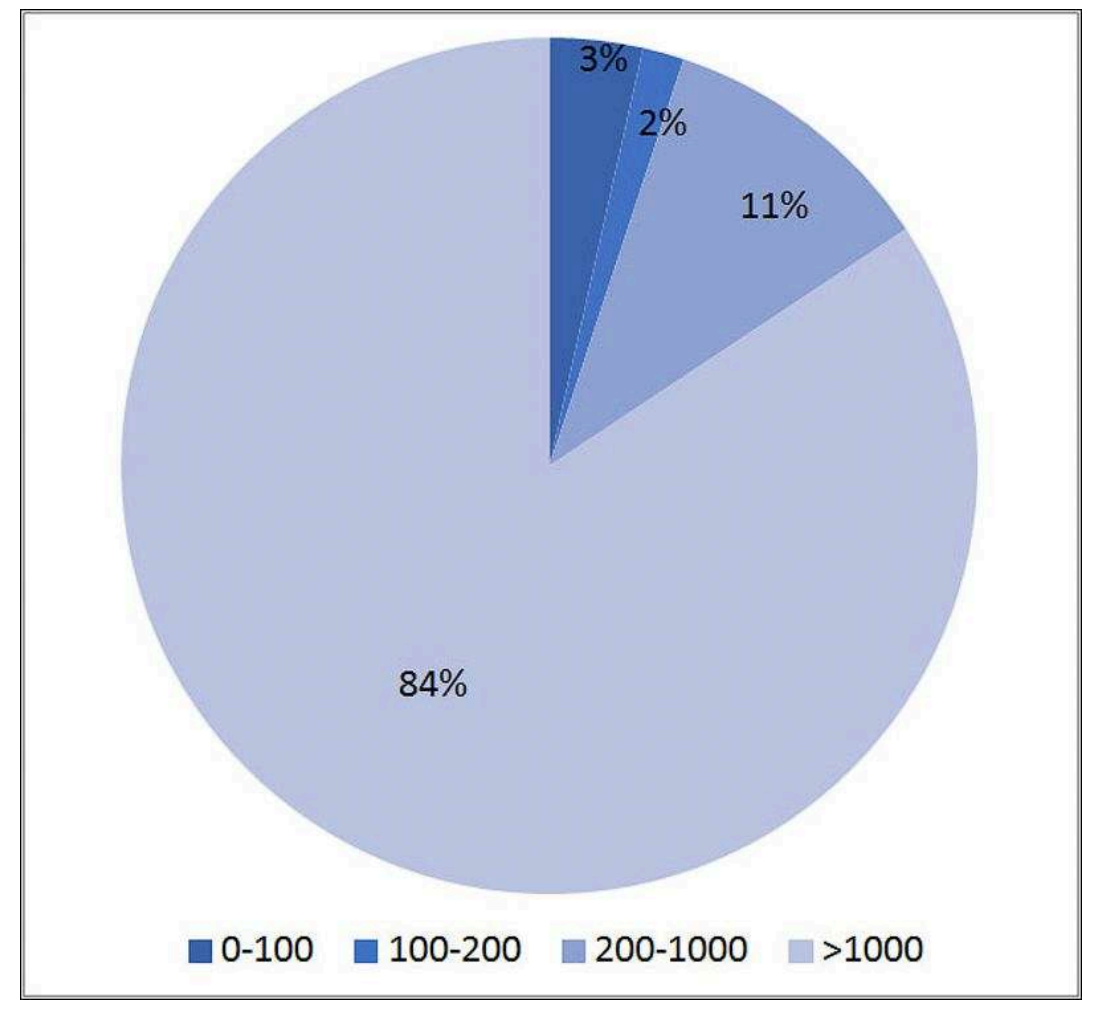

Source : SEMAS, 2016 - Brasília. Les premiers colons viennent surtout de la région centrale du Brésil, ainsi que l'indique le nom Para-Go-Minas (Pará, Goiás et Minas Gerais). Durant les années 1980, la municipalité est la première productrice de bois au Brésil du fait de l'exploitation massive des forêts primaires (Veríssimo et al., 1992). De 1983 à 1992, elle est également la première productrice de bovins de l'État du Pará et jusqu'en 2003, de charbon de bois (pour alimenter l'industrie sidérurgique à Marabá et au Maranhão) (Pinto et al., 2009). Les années 2000-2003 marquent le début de l'expansion de la culture de grains avec le riz, le maïs et le soja. Paragominas atteint en quelques années des volumes de production parmi les plus élevés de l'État du Pará. Ce développement s'accompagne d'une déforestation massive. Dès 2006, la municipalité est directement concernée par l'instauration du moratoire sur le soja avec plus de 5000 ha semés en soja (Piketty et al., 2015). En 2007, le gouvernement fédéral place la municipalité sur la liste noire. L'IBAMA y mène des opérations coup de poing exemplaires, conduisant à fermer les scieries et fours à charbon de bois illégaux, et un grand nombre de salariés se retrouvent au chômage (Piketty et al., 2015 ; Viana et al., 2016).

Dans ce contexte de crise, un tournant environnemental s'opère à Paragominas, qui résulte à la fois des régulations gouvernementales (fédérales et de l'État du Pará) et d'initiatives développées localement. Si la motivation première est de sortir de la liste noire et ainsi de l'embargo et des pénalités associées, les acteurs locaux cherchent également à rassurer les investisseurs et les acheteurs inquiets de l'insécurité générée par une image négative et par les boycotts des groupes de pression environnementalistes. Dès février 2008, les autorités municipales de Paragominas s'engagent avec les grands producteurs dans la démarche de Município Verde. C'est une 
première dans l'État du Pará. Un pacte contre la déforestation est d'abord signé entre la municipalité (Secrétariat de l'Environnement), les secteurs productifs (représentés par plusieurs syndicats des producteurs, de commerçants ou encore l'Association des producteurs de soja et de grains - Aprosoja) et l'Imazon, une ONG environnementaliste. Une coopération est également établie avec l'ONG The Nature Conservancy - TNC. Financé par l'entreprise minière Vale, le projet Paragominas: Município Verde se donne pour objectif de sortir de la liste noire et de créer les conditions de la régularisation environnementale des propriétés rurales notamment par le biais du suivi mensuel de la couverture forestière, de la formation de personnel pour le contrôle et la gestion environnementale et de l'éducation de la population municipale à l'environnement. Décrété comme instrument environnemental dès 2006 par l'État du Pará (Decreto Estadual 2.593/2006), le CAR devient à partir de 2010, grâce à la négociation du maire de Paragominas, l'une des deux conditions principales pour sortir de la liste noire (chaque municipalité devant cadastrer au moins $80 \%$ de sa surface privée), l'autre étant de réduire la déforestation à moins de $40 \mathrm{~km}^{2} / \mathrm{an}$ par municipalité.

Paragominas est la première municipalité d'Amazonie à sortir de la liste en 2010, avec plus de $95 \%$ du territoire privé enregistré dans le CAR grâce à l'appui de la TNC et de l'Imazon (Piketty et al., 2017). Cette sortie permet à nouveau l'accès au crédit pour les producteurs : en 2010, le Conselho Monetário Nacional (CMN) autorise les producteurs de Paragominas à emprunter, même en l'absence de titre de propriété, sous la réserve qu'ils aient entrepris des démarches de régularisation foncière et réalisé un CAR (Guimarães et al., 2011).

16 La réussite de ce projet municipal incite l'État du Pará à étendre en 2011 cette expérience avec le Programa Municípios Verdes (PMV). Le programme a pour objectif la réduction de la déforestation (de $80 \%$ d'ici à 2020), le retrait des municipalités de la liste noire, l'enregistrement des propriétés rurales dans le CAR et le renforcement de la gestion environnementale municipale afin de permettre une transition vers une production durable. Ce sont au total 107 municipalités du Pará qui ont adhéré au programme et qui bénéficient depuis 2014 d'un soutien financier de la part du Fonds Amazonie : près de 82,4 millions de Reais sur trois ans (Fundo Amazônia, 2017). Mené en partenariat avec les municipalités, la société civile, les initiatives privées, l'IBAMA et le Ministère Public Fédéral (MPF), le PMV est devenu le véritable point de départ d'une stratégie globale de l'État. Depuis 2008, en tant que membre du Groupe de Travail des Gouverneurs sur le Climat et les Forêts (GCF - Governors' Climate and Forests Task Force), l'État du Pará s'implique à investir et à développer des mesures innovantes pour freiner la déforestation. Récemment, il a signé la Déclaration de Rio Branco, s'engageant au côté de 26 membres (États, provinces) du GCF, à investir pour garantir la réduction de $80 \%$ des déboisements d'ici à 2020. Cette nouvelle inscription à l'agenda fait partie d'une stratégie qui vise à s'assurer que la croissance économique (à laquelle l'agriculture prend une part importante) s'opère dans un contexte environnemental favorable, une stratégie clairement reprise dans le plan stratégique appelé Pará 2030 lancé en 2016. La même année, le Pará s'est également distingué en étant le premier État amazonien à lancer son Plan de Régularisation Environnementale (PRA).

Ainsi, l'articulation entre initiatives municipales (e. g. Município Verde) et celles conduites au niveau des États (le Programa Municípios Verdes au Pará ou le Programa Mato Grosso MT - Legal inspiré de l'expérience de la municipalité de Lucas do Rio Verde) 
permet une mise en cohérence des politiques environnementales entre échelons administratifs.

\section{Vers un découplage entre production agricole et déforestation}

Que ce soit au niveau fédéral, fédéré ou local, les institutions politiques ont engagé un tournant environnemental, au travers de nouvelles régulations et programmes visant une réduction de la déforestation. Différents indicateurs attestent de la baisse de la déforestation et montrent qu'elle a induit une transformation du secteur agricole, qui passe d'une logique d'expansion à une logique d'intensification et de diversification.

\section{L'intensification et la diversification au cœur du nouveau modèle de développement agricole en Amazonie}

Après une forte croissance de la déforestation en Amazonie au cours des années 1990, la mise en place de politiques publiques efficaces dans le cadre du PPCDAm a permis de diminuer les taux de déforestation à partir de 2005 (illustration 4). D'après les données PRODES de l'INPE, ces taux ont baissé de $77 \%$ entre 2004 et 2015, passant de $26859 \mathrm{~km}^{2}$ à $5949 \mathrm{~km}^{2}$ par an dans la région Nord et au Mato Grosso ${ }^{11}$ (INPE, 2016). La chute du taux de déforestation est dans un premier temps marquée par un recul des surfaces cultivées et des effectifs bovins entre 2005 et 2007. Cette période de flottement s'explique par une difficulté d'adaptation des producteurs aux nouvelles contraintes environnementales mais aussi par une forte valorisation de la monnaie brésilienne (R\$) par rapport au dollar (US\$) freinant les exportations de produits agricoles. Après 2007, la phase d'adaptation semble terminée. La surface annuellement déforestée continue de chuter tandis que les surfaces cultivées en soja et les effectifs bovins repartent à la hausse (illustration 4), témoignant d'un profond changement dans les relations entre développement agricole et environnement en Amazonie.

L'élevage bovin en Amazonie connaît une intensification fondée principalement sur le mode de gestion des prairies : sélection des espèces de graminées implantées, réduction des temps de pâture afin de diminuer la compaction des sols par l'installation de clôtures, correction de l'acidité des sols par des amendements calcaires et apport de fertilisants phosphorés (Poccard-Chapuis et al., 2015). Des formes d'intensification plus poussées sous forme d'élevage hors sol sont quasiment inexistantes en Amazonie. 
Illustration 4 - Évolution de la déforestation, des surfaces cultivées en soja et des effectifs bovins en Amazonie (région Nord et Mato Grosso) et à Paragominas entre 1990 et 2015

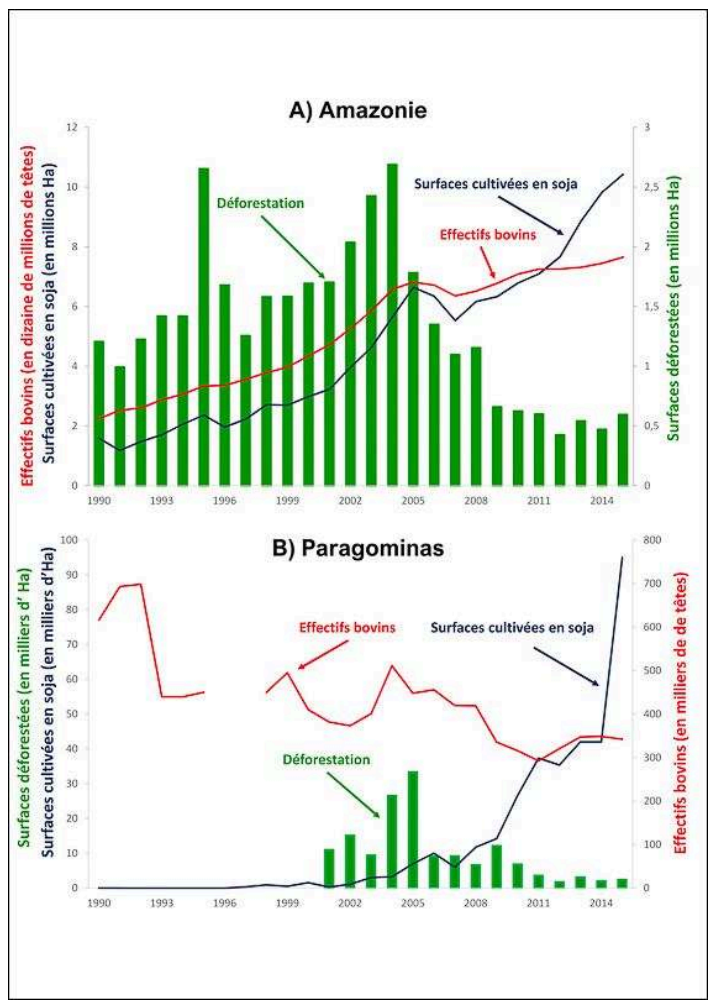

Les données de déforestation à Paragominas issues du PRODES ne sont disponibles qu'à partir de 2000.

Sources : INPE, 2016 et IBGE, 2017b.

21 En ce qui concerne le soja, la forte croissance de la filière s'explique principalement par l'adoption de nouvelles pratiques agricoles qui ont pris le pas sur le processus d'expansion par déforestation pour accroître la production. Ainsi, les années 2000 correspondent à une phase d'intensification et de diversification agricole symbolisée par la généralisation du semis direct (SD) et des systèmes de double-cultures (DC), c'està-dire une culture de maïs, coton, sorgho ou millet étant semée après la récolte de soja. Dans l'État du Mato Grosso, les surfaces en DC sont par exemple passées de $35 \%$ à $62 \%$ du total des surfaces cultivées entre 2000 et 2007 (Arvor et al., 2012 ; Arvor et al., 2013). Quant au semis direct, la CONAB (Companhia Nacional de Abastecimento) estime qu'il concerne jusqu'à $90 \%$ des surfaces cultivées en soja dans certaines municipalités amazoniennes. Ces pratiques (DC et $\mathrm{SD}$ ) s'intègrent également dans une stratégie de diversification de la production qui permet de réduire la vulnérabilité de la filière agricole en période de crise, comme ce fut le cas sur la période 2005-2007. Ce processus de diversification s'accompagne actuellement d'un processus de verticalisation visant à transformer sur place les matières premières produites. De plus, les élevages de poissons, de volailles et de porcs se multiplient en Amazonie sous l'impulsion de l'installation de grands groupes agro-alimentaires (BRF, Perdigão, etc.) (Bonaudo et al., 2015 ; Arvor et al., 2016). Cependant, ces nouvelles activités soulèvent de nouveaux problèmes environnementaux (par exemple l'augmentation de l'utilisation de produits chimiques de 3,8 à 6,7 kg d'ingrédients actifs par hectare au Mato Grosso, l'insuffisante longueur des rotations du fait de deux à trois cultures principales, impliquant le recours abondant aux produits phytosanitaires), ce qui pose la question d'une 
transition agricole plus respectueuse de l'environnement (Arvor et al., 2017). Pour accompagner ces évolutions, le gouvernement brésilien a lancé en 2010 le Plan pour une agriculture à faible émission carbone (Plano $A B C$ ), qui comprend six programmes visant à soutenir l'adoption de pratiques d'agriculture de conservation. Parmi les principales techniques promues, on retrouve les pratiques de semis direct mais aussi la fixation de l'azote par des légumineuses, les systèmes de culture intégrés (l'objectif étant d'atteindre un total de 4 millions d'hectares cultivés en systèmes intégrés en 2020 , soit $6 \%$ de la superficie cultivée nationale totale) et la restauration des pâturages dégradés (Strassburg et al., 2014). Mais comme l'obtention des crédits $A B C$ exige différentes conditions et que leurs taux ne sont pas suffisamment attractifs, ils restent faiblement diffusés dans le biome amazonien.

Par ailleurs, le secteur agricole intègre peu à peu les nouvelles normes environnementales: recyclage des emballages de produits phytosanitaires, licences environnementales, respect du Code Forestier. D'abord réticents, les secteurs élevage et cultures se sont progressivement organisés, avec le soutien mais aussi sous la pression des ONG, et ont mis en place des programmes intégrant la question de la conservation des sols, de l'intensification de la production et de la préservation des cours d'eau (APP) et réserves légales (RL). Par exemple, en 2009 est constituée une Table Ronde sur l'Élevage Responsable au Brésil (GTPS) qui réunit de nombreuses parties prenantes (producteurs, industries, commerces et services, organisations de la société civile, établissements de recherche et universitaires, et acteurs institutionnels tels que le Ministère de l'Environnement - MMA) afin de promouvoir des systèmes de production plus durables en relayant des expériences. Signe d'un retour de confiance des marchés, le groupe McDonald's qui n'achetait plus de viande amazonienne depuis une décennie, a repris l'achat de bétail provenant de fermes partenaires du programme Novo Campo de la municipalité d'Alta Floresta (Mato Grosso) (ICV, 2016). En 2006 déjà, une démarche similaire au GTPS avait été développée dans le secteur des grandes cultures avec la création de la Table Ronde du Soja Responsable (RTRS).

\section{À Paragominas, expansion du soja et baisse de la déforestation}

23 Les données PRODES sur la municipalité de Paragominas montrent des taux de déforestation élevés jusqu'en 2005 (perte moyenne de 19080 ha/an, de 2001 à 2005), puis un premier palier de baisse de 2006 à 2010 (perte moyenne de $8780 \mathrm{ha} / \mathrm{an}$ ) et un net recul de la déforestation de 2011 à 2015 (perte moyenne de 2570 ha/an) (INPE, 2016) (illustration 4). Les surfaces en soja augmentent fortement en 2010 et en 2011 puis en 2015, alors que la déforestation baisse. L'effectif bovin recule de 2004 à 2009 puis se stabilise. L'augmentation des surfaces en soja à partir de 2010 ne s'est donc traduite ni par une baisse des effectifs bovins, ni par une déforestation. Cela en évidence le découplage entre déforestation et production agricole et indique une intensification accompagnant la diversification: sur un même espace ouvert, la production de soja augmente sans réduire la production bovine. Le soja est de plus en plus cultivé en semis direct afin de réduire les coûts de production et l'érosion des sols (Alves et al., 2014).

24 La limitation de la déforestation se traduit par une réorganisation de l'espace auparavant déforesté. Une réallocation des usages des sols en fonction des aptitudes agronomiques s'observe notamment. L'extension des surfaces en cultures annuelles 
comme le soja ou le maïs privilégie les sols les plus fertiles tandis que le critère prépondérant pour l'élevage est l'accès à l'eau pour l'abreuvement du bétail. Bien que globalement plus réduite à présent, la déforestation qui visait auparavant les fonds de vallées offrant un accès aux cours d'eau se concentre maintenant sur les sols argileux de plateau plus fertiles pour permettre l'expansion des cultures annuelles. La part des sols argileux de plateau dans les surfaces déforestées est ainsi passée de $20 \%$ en 2001 à 70 \% en 2013 (Piketty et al., 2015).

Un autre facteur expliquant la localisation des surfaces mises en cultures est la proximité des routes goudronnées. Ce facteur est particulièrement important dans les systèmes intensifs où l'application des intrants, le semis et la récolte nécessitent un accès pour les engins agricoles. Une analyse spatiale de l'occupation du sol selon les données Terraclass en 2010 montre que les zones à moins de $20 \mathrm{~km}$ des grandes routes représentent $23 \%$ de la surface municipale mais concentrent $78 \%$ des surfaces en cultures annuelles contre seulement $10 \%$ des forêts natives (Piketty et al., 2015). Dans ces zones, les cultures annuelles se développent souvent sous forme intégrée à l'élevage, permettant de restaurer les pâturages dégradés à moindre coût (PoccardChapuis et al., 2015). Environ $60 \%$ des sojiculteurs louent les terres de grands éleveurs, souvent pour des contrats de 6 ans, durée après laquelle ceux-ci peuvent semer les surfaces en pâturages intensifs bénéficiant alors des reliquats de fertilisation et de l'élimination des ligneuses invasives.

Les données de l'IBGE (IBGE, 2017b) montrent une baisse tendancielle du nombre de têtes de bovins de 2004 à 2015 (de 511000 à 342000 têtes) et une très forte expansion des surfaces en soja (de 3300 ha à 95000 ha) à Paragominas (illustration 4), tandis que sur l'ensemble de l'Amazonie, le nombre de bovins augmente et que les surfaces en soja ont doublé sur la même période. Cette différenciation de Paragominas révèle une intensification et une diversification plus amples que dans le reste du bassin amazonien, de par la conjonction d'un certain nombre de facteurs: les producteurs locaux avancent la proximité des ports d'exportation qui réduit les coûts de transport, l'organisation de la filière soja sur la ville avec des services associés à la production, mais des facteurs climatiques y participent également. En effet, le remplacement des pâturages par des cultures annuelles s'accentue d'autant plus dans cette région du fait de saisons sèches plus longues et sévères que dans le reste de l'Amazonie. Des évènements de sécheresse intense, comme ce fut le cas en 2005, 2006, 2015 et 2016 ne font que favoriser ce processus. Les cultures annuelles étant pratiquées en saison des pluies, leurs rendements ne sont pas affectés par l'intensité de la saison sèche (dans cette région, une seule culture est le plus souvent pratiquée, la durée de la saison des pluies étant insuffisante pour une seconde culture). À l'inverse, l'élevage nécessite une production continue de fourrages en l'absence d'ensilage ou de fanage. Les sécheresses répétées fragilisent donc l'élevage au profit des cultures annuelles ou de la sylviculture et participent, parmi bien d'autres facteurs, au recul de l'élevage et à la diversification.

\section{Un tournant environnemental qui laisse les agriculteurs familiaux à l'écart}

La baisse de la déforestation et la croissance du secteur agricole sont la preuve de la capacité d'adaptation des filières à l'évolution des politiques publiques en Amazonie. Cependant les écarts se creusent entre d'une part, les moyens et grands producteurs 
intégrés aux marchés et se conformant aux législations environnementales, et d'autre part, les agriculteurs familiaux qui n'arrivent pas à s'adapter et qui sont exclus du tournant environnemental. Ceux-ci deviennent encore plus vulnérables. La sortie de cette spirale implique une meilleure prise en compte des contraintes et des logiques de l'agriculture familiale par les pouvoirs publics.

\section{Une inégale sortie de la déforestation}

Les relations entre déforestation et croissance économique en Amazonie ont changé au cours du temps. À la fin des années 1990, la déforestation s'inscrit dans une vision à court terme, orientée vers une croissance économique rapide, consommatrice d'espace ("géophagique" pour reprendre le terme de Droulers, 2001) aux dépens des ressources naturelles. C'est principalement l'œuvre de grands propriétaires ou cela sert à terme les grands propriétaires, comme l'a montré particulièrement Xavier Arnauld de Sartre (2005) : la dynamique migratoire à l'origine de l'ouverture de fronts pionniers par de petits producteurs s'appuie sur le besoin d'acquérir du foncier avant de le revendre à de grands propriétaires. À la fin des années 2000, comme mentionné précédemment, on assiste à un découplage entre déforestation et production agricole qui s'accompagne de l'instauration d'une relation de type Environmental Kuznets Curve (EKC) entre déforestation et revenu des ménages (illustration 5). Sur la période 1997-2000, les espaces où les revenus sont élevés sont bien les plus actifs en termes de déforestation. Un tournant s'opère alors et une courbe en U inversé, caractéristique de EKC, émerge à la fin des années 2000 : les espaces à hauts revenus sont moins déboisés que dans les espaces où les revenus sont plus faibles (autour d'un salaire minimum). On note ainsi l'apparition de zones de post-frontière relativement prospères où la déforestation est stabilisée alors que les zones de frontière où la déforestation persiste encore en 2010 sont marquées par des indicateurs économiques plus faibles (Tritsch et Arvor, 2016). La déforestation peut dès lors être associée à une certaine précarité économique et devient un marqueur des inégalités en Amazonie. 
Illustration 5. Évolution des taux de déforestation entre 2000 et 2010 en fonction des revenus par foyer

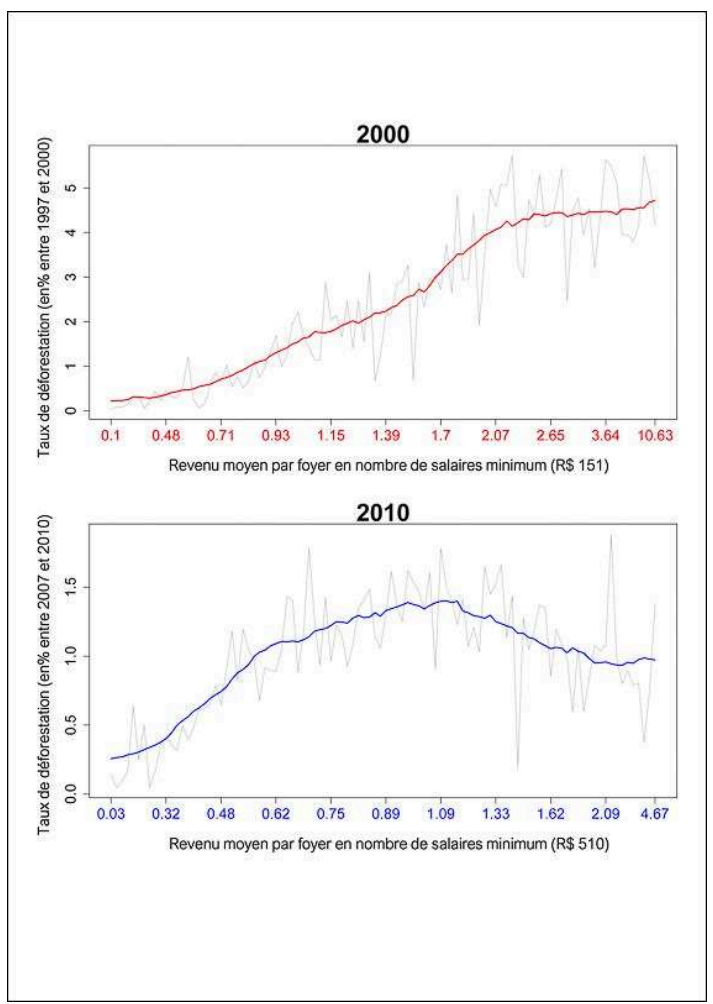

Les taux de déforestation pour 2000 et 2010 sont calculés à partir des données PRODES (INPE, 2016) sur les périodes 1997-2000 et 2007-2010, respectivement, pour des cellules de 10*10 km ordonnées en fonction de leur revenu moyen par foyer (calculé à partir des données IBGE des recensements de 2000 et 2010 à l'échelle des secteurs censitaires; IBGE, 2010).

Source : Tritsch et Arvor, 2016.

À Paragominas, des écarts socio-économiques s'observent également sur la question de la déforestation. En effet, la dynamique actuelle d'adaptation aux règles environnementales inclut difficilement les agriculteurs familiaux qui subissent moins la pression des filières, ne disposent pas de moyens matériels pour entretenir les pâturages ou cultiver sans le feu, ou n'ont pas accès aux connaissances de pratiques alternatives (Coudel et al., 2012; Cialdella et al., 2015 ; Piketty et al., 2017). Cela est mis en évidence par les différences d'évolution de l'utilisation du sol selon la taille des propriétés (illustration 6). Sur la période allant de 2004 à 2007, la part de forêt (sans pouvoir distinguer les forêts primaires, des forêts secondaires ou des plantations sylvicoles d'essences commerciales) a reculé dans toutes les classes de propriété, mais plus rapidement dans les petites exploitations que dans les grandes. De 2007 à 2015, la forêt a continué de reculer dans les exploitations de 20 à 200 ha tandis qu'elle s'est étendue dans les moyennes et grandes exploitations (plus de 200 ha). En 2015, la part en forêt avoisine $70 \%$ dans les propriétés de plus de 1000 ha, tandis qu'elle est inférieure à $50 \%$ dans les classes inférieures. L'accroissement récent des surfaces en forêt vient à la fois de la récupération partielle de la dette environnementale de certains producteurs, mais aussi de l'extension de la sylviculture de plantation à base d'espèces commerciales comme l'eucalyptus et le paricá (Schizolobium amazonicum) sur des pâturages dégradés. En ce qui concerne les cultures, elles ont progressé dans toutes les classes sur les deux périodes, mais de 2007 à 2015 leur extension a été plus rapide dans 
les moyennes et dans les grandes exploitations où leur surface a triplé. Les pâturages se sont étendus de 2004 à 2007 dans toutes les exploitations puis ont systématiquement reculé dans la période suivante. Ces évolutions peuvent s'expliquer par le fait que depuis 2007, les moyennes et grandes exploitations cherchent à se mettre en conformité vis-à-vis de la réglementation sur la réserve légale (sans pour autant atteindre les $80 \%$ ) tout en diversifiant leurs productions, notamment vers les cultures annuelles comme le soja, dont les prix ont augmenté depuis 2007, ou vers la sylviculture de plantation. En revanche, dans les petites exploitations familiales, l'accroissement des surfaces cultivées continue de s'accompagner de déforestation.

Illustration 6 - Évolution de l'occupation du sol à Paragominas en fonction de la taille des propriétés

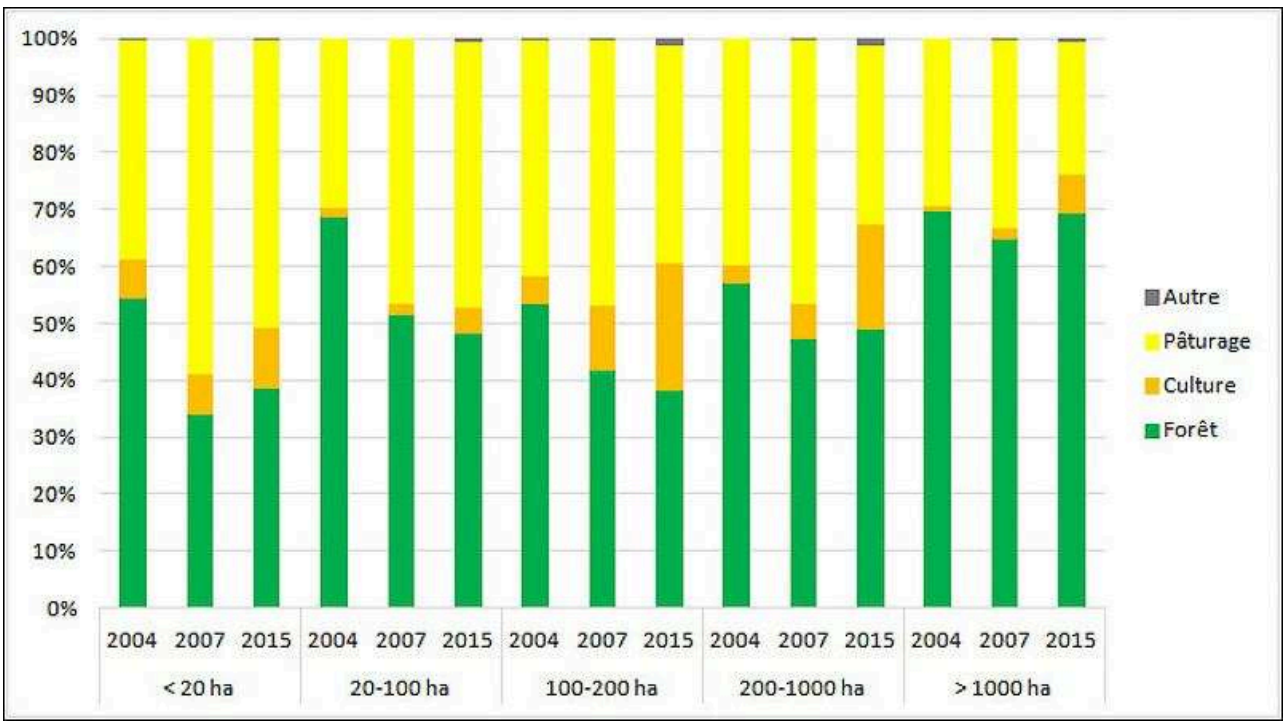

Sources des données : SEMAS (2016), classifications d'occupation du sol opérées sur des images MODIS par Perrier F. (2014).

\section{Vers une meilleure prise en compte des agriculteurs familiaux ?}

La dynamique de l'occupation du sol à Paragominas montre que les évolutions actuelles laissent souvent de côté les agriculteurs familiaux, ce qui remet en cause la durabilité $\mathrm{du}$ développement des territoires amazoniens. Sans politiques complémentaires, la "zéro déforestation" prônée par les instances publiques à différents niveaux fragilise ceux qui n'ont pas les moyens d'abandonner les pratiques de culture sur brûlis aux dépens des ressources forestières ou de ne plus recourir au feu pour nettoyer les pâturages. En outre, malgré la mise en place de plusieurs programmes sociaux dans beaucoup de territoires amazoniens, de très fortes asymétries entre l'agriculture familiale et l'agrobusiness persistent, ce qui freine la participation de l'agriculture familiale à la gouvernance territoriale et donc la mise en place de mesures adaptées à leurs besoins (Piraux et al., 2015).

31 À Paragominas, différentes initiatives ont cherché à étendre la dynamique Município Verde aux agriculteurs familiaux, mais souvent au travers d'une vision menée par l'agrobusiness, peu adaptée à la réalité des agriculteurs familiaux (Carneiro et Assis, 2015 ; Viana et al., 2016). Ainsi, des patrouilles de tracteurs ont été proposées pour labourer les terres des agriculteurs familiaux, mais sans penser à la manière dont se 
ferait l'apport de fertilisants et avec des capacités limitées (au meilleur moment, 12 tracteurs pour plus de 5000 agriculteurs familiaux). Une intégration à un ramassage laitier a été proposée dans la zone la plus touchée par la maladie du charbon, pour diversifier l'économie de la communauté, mais les conditions des pâturages permettaient une production tellement faible que le ramassage quotidien n'était pas viable. Une autre initiative est la création d'un abattoir, à l'initiative des principaux grands producteurs bovins, qui a profité de financements spécifiques pour l'agriculture familiale en faisant valoir qu'il pourrait servir aussi pour les porcins et caprins. Ces producteurs souhaitaient également valoriser ainsi localement la production de soja et de maïs, mais le modèle technique proposé aux agriculteurs intéressés est de plus de 100 matrices porcines, c'est-à-dire un modèle industriel qui ne correspond pas à la réalité de l'agriculture familiale actuelle et qui suppose un endettement conséquent.

L'initiative la plus fructueuse est celle des cantines scolaires, en lien avec le programme d'alimentation scolaire (PNAE) du gouvernement fédéral. Chaque municipalité est censée se fournir localement pour $30 \%$ des produits offerts aux cantines municipales. La municipalité de Paragominas a reçu 8 ans d'affilée le prix du meilleur programme, mettant ainsi en place des filières courtes de produits maraîchers autour de la ville mais aussi dans certaines des plus grosses communautés d'agriculteurs familiaux. Mais ceci concerne pour le moment seulement une vingtaine d'agriculteurs et la grande majorité reste frustrée par ces programmes ponctuels qui ne leur sont pas adaptés et qui ne sont pas suffisants pour permettre une réelle transformation de leurs pratiques.

Bien que l'appui à la production soit encore diffus au niveau fédéral, avec une assistance technique et des programmes de crédits qui peinent à se restructurer, différentes mesures ont été prises pour faciliter la mise aux normes environnementales des agriculteurs familiaux. Si la réforme du Code Forestier de $2012 \mathrm{a}$ fortement assoupli les règles en termes de $\mathrm{CAR}$ et de reboisement des terres illégalement déforestées pour les agriculteurs familiaux, leur manque de ressources financières ou techniques pour se mettre en conformité implique des difficultés supplémentaires. Plusieurs initiatives privées (ONG) et publiques (projets municipaux) ont d'ailleurs vu le jour ces dernières années en Amazonie (notamment dans les États du Pará et du Mato Grosso) pour permettre la régularisation environnementale des agriculteurs familiaux. La mise en œuvre de la récupération environnementale mentionnée dans le Code Forestier est abordée dans le Plano Nacional de Recuperação da Vegetação Nativa (MMA, 2014) et, plus récemment, en 2017, dans la Política Nacional de Recuperação da Vegetação Nativa (BRASIL, 2017) censée faire appliquer ce plan. Il souligne l'importance des instruments prévus par la loi afin de soutenir cette mise en conformité environnementale à travers l'accès à des paiements pour services environnementaux (PSE), des dons, des crédits et des financements.

Néanmoins, comme l'indique le délai entre le plan et le décret, la mise en place concrète de ces modalités au niveau fédéral est longue (Coudel et al., 2015). Les avancées au niveau local, en particulier municipal ou territorial, peuvent être bien plus rapides. Des projets territoriaux négociés entre acteurs publics et société civile à ce niveau permettent aussi de construire des réponses adéquates et adaptées aux réalités locales des agricultures familiales. Mais il est alors nécessaire de renforcer les capacités locales et des dynamiques collectives. Le travail de prospective territoriale mené dans le cadre $\mathrm{du}$ projet ECOTERA poursuit cet objectif : aider les agriculteurs familiaux à mieux se représenter les problèmes globaux et locaux de la municipalité, comprendre les 
facteurs qui peuvent jouer dans la consolidation de ce secteur dans le futur, et définir les grands axes d'un projet à négocier avec les partenaires publics. Les pouvoirs publics, en particulier les municipalités, ont en effet un rôle important à jouer pour permettre la concertation locale nécessaire à l'identification de pratiques alternatives, de besoins en financement et d'appuis techniques adaptés (Tonneau et al., 2009).

\section{Conclusion}

Le tournant environnemental en Amazonie s'opère à plusieurs échelles et se manifeste par de profondes transformations dans l'usage de l'espace et des ressources naturelles par l'agriculture. Il résulte de la combinaison de mesures mises en œuvre pour freiner la déforestation, des changements des systèmes de production et des pratiques agricoles et de programmes territoriaux se réclamant de la durabilité (Município Verde, pecuária sustentável, soja verde, crescimento sustentável, etc.). Le secteur agricole s'est adapté sous la contrainte du Code Forestier et de la pression des consommateurs relayée par les filières. Nombre de producteurs ont intégré le nouveau contexte et sont passés d'une stratégie d'expansion à une stratégie d'intensification et de diversification. Observée aux échelles locales et régionales, la déforestation a ainsi fortement baissé jusqu'en 2014. Paragominas illustre ces évolutions en combinant une profonde transformation de l'espace agricole (avec l'expansion du soja et de la sylviculture) à une réduction importante de la déforestation.

36 Pourtant, des écarts importants persistent selon la taille des propriétés et le type d'agriculteurs. L'agrobusiness arrive à s'adapter au nouveau cadre normatif : le secteur étant très organisé et capitalisé, il adopte des pratiques ne nécessitant plus de déforestation (bien que la réalité soit moins systématique et plus complexe). Globalement, la croissance des revenus des moyens et grands producteurs n'est plus synonyme de déforestation comme l'atteste le découplage entre production agricole et déforestation. Par contre, ces adaptations sont plus difficiles pour les agriculteurs familiaux, qui ont peu d'alternatives à la culture sur brûlis, à l'utilisation du feu pour le nettoyage des pâturages, et ne disposent pas des équipements ou des intrants qui permettraient une transition. Ainsi, l'agriculture familiale intègre difficilement la dynamique amorcée en 2005. À Paragominas, la forêt continue de reculer dans les petites propriétés, alors qu'elle s'étend dans les moyennes et dans les grandes.

La reprise des déboisements sur deux années successives à l'échelle de l'Amazonie légale ( $+24 \%$ en 2015 et $+29 \%$ en 2016 selon l'INPE, 2016) et l'ampleur des incendies qui ont sévi posent la question de la maîtrise de la déforestation (Le Tourneau, 2015) et montrent la fragilité du tournant environnemental. Azevedo et al. (2015 et 2017) ont souligné à travers l'exemple de l'État du Mato Grosso que la mise en conformité des propriétés et le CAR ne vont pas forcément de pair avec l'arrêt de la déforestation, une observation que corroborent les résultats publiés par la Folha de São Paulo (Watanabe, 2017) et issus des analyses de l'Imazon: $56 \%$ des terres déboisées en 2016 (en grande majorité illégalement), l'ont été au sein de propriétés enregistrées dans le CAR. Le manque de contrôles et de sanctions liés à la crise politique actuelle, couplé à la lente mise en place des instruments prévus par la réforme du Code Forestier, ou encore à l'incertitude qui règne sur la question de la régularisation foncière (accaparement des terres, spéculation foncière) sont mises en avant pour expliquer cette reprise de la déforestation après dix années de baisse. 
, la dégradation de l'environnement ne se réduit pas à la seule déforestation. D'autres indicateurs méritent d'être développés pour juger de la réussite du tournant environnemental. En effet, les forêts sont soumises à un processus de dégradation forestière, c'est à dire à une transformation de leur structure, une réduction de leur biodiversité et à une perte concomitante de leurs fonctionnalités écologiques, réduisant ainsi les services écosystémiques qu'elles fournissent. La dégradation forestière résulte de l'exploitation du bois et d'incendies plus ou moins fréquents (Gerwing, 2002 ; Tritsch et al., 2016; Richardson et al., 2016). À titre indicatif, entre 2000 et $2010,50815 \mathrm{~km}^{2}$ de forêt ont été dégradés en Amazonie brésilienne, ce qui équivaut à $30 \%$ de la superficie convertie par la déforestation sur la même période (Souza et al., 2013). De plus, cette même étude montre que si la déforestation a fortement diminué après 2006, la dégradation forestière a, au contraire, augmenté d'environ $20 \%$. Dans ce contexte, l'évaluation du tournant environnemental en Amazonie devrait intégrer ce processus.

\section{BIBLIOGRAPHY}

Alves LW.R., Carvalho E.J.M., Silva L.G.T., 2014. Diagnóstico agrícola do município de Paragominas, PA /- Belém, PA. Embrapa Amazônia Oriental, 26 p.

Arima E.Y., Barreto P., Araujo E., Soares-Filho B., 2014. Public policies can reduce tropical deforestation: Lessons and challenges from Brazil. Land Use Policy, 41, p. 465-473. DOI : 10.1016/ j.landusepol.2014.06.026

Arnauld de Sartre X., 2005. Modernité et développement durable. Le cas des fronts pionniers d'Amazonie Orientale. Espaces et sociétés, 2005/1, n 120-121, p. 219-239. DOI : 10.3917/esp. 120.0219

Arvor D., Daugeard M., Tritsch I., de Mello-Théry N.A., Théry H., Dubreuil V., 2016. Combining socio-economic development with environmental governance in the Brazilian Amazon: The Mato Grosso agricultural frontier at a tipping point. Environment Development and Sustainability, p. 1-22. DOI : $10.1007 /$ s10668-016-9889-1

Arvor D., Dubreuil V., Meirelles M.S.P., Bégué A., 2013. Mapping and spatial analysis of the soybean agricultural frontier in Mato Grosso, Brazil, using remote sensing data. GeoJournal, 78(5), p. 833-850. DOI : 10.1007/s10708-012-9469-3

Arvor D., Meirelles M. S. P., Dubreuil V., Shimabukuro Y. E., Bégué A., 2012. Analysing the agricultural transition in Mato Grosso, Brazil, using satellite-derived indices. Applied Geography, 32, 2, p. 702-713.

Arvor D., Tritsch I., Barcellos C., Jégou N., Dubreuil V., 2017. Land use sustainability on the SouthEastern Amazon agricultural frontier: Recent progress and the challenges ahead. Applied Geography, 80, p. 86-97. DOI : 10.1016/j.apgeog.2017.02.003

Azevedo A. A., Rajão R., Costa M. A., Stabile M. C. C., Macedo M. N., Reis T. N. P., Pacheco R., 2017. Limits of Brazil's Forest Code as a means to end illegal deforestation. Proceedings of the National Academy of Sciences, 6. https://doi.org/10.1073/pnas.1604768114 
Azevedo A. A., Stabile M. C. C., Reis T. N. P., 2015. Commodity production in Brazil: Combining zero deforestation and zero illegality. Elementa: Science of the Anthropocene, 3(1), 12. https:// doi.org/10.12952/journal.elementa.000076

Barreto P., Silva D., 2010. Will Cattle Ranching Continue to Drive Deforestation in the Brazilian Amazon? Presented at the Environment and Natural Resources Management in Developing and Transition Economies, CERDI, Clermont-Ferrand, France. http://cerdi.org/uploads/sfCmsContent/ html/323/Barreto.pdf

Bonaudo T., Poccard-Chapuis R., Coutinho C., Tourrand J.F. et Duarte L., 2015. Le cluster de Rio Verde ou l'interaction de l'agrobusiness et du développement territorial au Brésil. Confins, 25. DOI : 10.4000/confins.10506

Brannstrom C., Rausch L., Brown J.C., de Andrade R.M.T., Miccolis A., 2012. Compliance and market exclusion in Brazilian agriculture: Analysis and implications for "soft" governance. Land Use Policy, 29, 2, p. 357-366. DOI : 10.1016/j.landusepol.2011.07.006

BRASIL, 2017. Decreto $n^{\circ}$ 8.972, de 23 de Janeiro de 2017, Institui a Política Nacional de Recuperação da Vegetação Nativa. http://www.planalto.gov.br/ccivil_03/_ato2015-2018/2017/decreto/D8972.htm

BRASIL, 2012. Lei no 12.651, de 25 de Maio de 2012, Dispõe sobre a proteção da vegetação nativa. http:// www.planalto.gov.br/ccivil_03/_ato2011-2014/2012/lei/l12651.htm

Butler R., Laurance W., 2008. New strategies for conserving tropical forests. Trends in Ecology \& Evolution, 23, 9, p. 469-472. DOI :10.1016/j.tree.2008.05.006

Carneiro M. S., de Assis W. S., 2015. O controle do desmatamento na Amazônia como um processo de modernização ecológica: a experiência do Projeto Município Verde. Revista Pós Ciências Sociais, 12, 24, p. 53-76. http://dx.doi.org/10.18764/2236-9473.v12n24p53-76

Cialdella N., Carvalho S.A, Vaz V., Barbosa T., Thâles M., Mourão M., Poccard-Chapuis R., Tourrand J.F., 2015. Do political changes aimed at reducing Amazonian deforestation contribute to ecological intensification? Cahiers Agricultures, 24, p. 246-254. DOI : http://dx.doi.org/10.1684/ agr.2015.0761

Coudel E., Ferreira J., Amazonas M., Eloy L., Hercowitz M., Mattos L., May P., Muradian R., Piketty M.G., Toni F., 2015. Chapter 18: The rise of PES in Brazil: from pilot projects to public policies. In Martínez-Alier J., Muradian R., Handbook of Ecological Economics. Chentelham, UK, Edward Elgar, p. $450-472$.

Coudel E., Piketty M.G., Gardner T.A., Viana C., Ferreira J.N., Morello T., Parry L., Barlow J., Antona M., 2012. Environmental compliance in the Brazilian Amazon: exploring motivations and institutional conditions. In 12th Biennial Conference of the International Society for Ecological Economics (ISEE 2012 Conference), Ecological Economics and Rio+20: Challenges and Contributions for a Green Economy, 16-19 June 2012, Rio de Janeiro, Brazil, p. 34.

Coy M., Klingler M., Kohlhepp G., 2017. De frontier até pós-frontier: regiões pioneiras no Brasil dentro do processo de transformação espaço-temporal e sócio-ecológico. Confins, 30 | 2017, mis en ligne le 20/02/2017, consulté le 22/03/2017. http://confins.revues.org/11683 ; DOI : 10.4000/ confins. 11683

De Mello N. A., Théry, H., 2003. L'État brésilien et l'environnement en Amazonie : évolutions, contradictions et conflits. Espace Géographique, 32, 3, p. 3-20. DOI : 10.3917/eg.321.0003

Drigo I., 2013. Towards sustainable and certified beef? The reasons why and mechanisms through beef producers are beginning to change their production practices. Final Post-doc report, Sao Paulo University, São Paulo, Brazil, 88 p. 
Droulers M., 2001. Le Brésil: une géohistoire. Presses Universitaires de France, 306 p.

Dubreuil V. (dir.), 2002. Environnement et télédétection au Brésil. Éditions Presses Universitaires de Rennes. 202 p.

Fundo Amazônia, 2017. Projetos Apoiados. Programa Municípios Verdes, http:// www.fundoamazonia.gov.br/FundoAmazonia/fam/site_pt/Esquerdo/Projetos_Apoiados/ Lista_Projetos/Estado_Para_PMV, consulté le 20/02/2017.

Gibbs H.K., Munger J., L’Roe J., Barreto P., Pereira R., Christie M., Amaral T., Walker N.F., 2016. Did Ranchers and Slaughterhouses Respond to Zero-Deforestation Agreements in the Brazilian Amazon? Conservation Letters, 9, 1, p. 32-42. DOI :10.1111/conl.12175

Gibbs H.K., Rausch L., Munger J., Schelly I., Morton D.C., Noojipady P., Soares-Filho B., Barreto P., Micol L., Walker N.F., 2015. Brazil's Soy Moratorium. Science, 347, p. 377-378. DOI :10.1126/ science.aaa0181

Gerwing, J. J., 2002. Degradation of forests through logging and fire in the eastern Brazilian Amazon. Forest ecology and management, 157, 1, p. 131-141.

Greenpeace International, 2006. Eating Up the Amazon. Amsterdam, NL, 64 p. http:// www.greenpeace.org/international/Global/international/planet-2/report/2006/7/eating-upthe-amazon.pdf

Greenpeace International, 2009. Slaughtering the Amazon. Amsterdam, NL, 34 p. http:// www.greenpeace.org/international/Global/international/planet-2/binaries/2009/7/ slaughtering-the-amazon-part1.pdf

Guimarães J., Veríssimo A., Amaral, P. Demachki A., 2011. Municípios Verdes: caminhos para a sustentabilidade. IMAZON, Belém, Brazil, $154 \mathrm{p}$.

IBGE, 2010. Base de données "setores censitários". ftp://ftp.ibge.gov.br/Censos/, consulté le 01/03/2015.

IBGE, 2017a. Base de données “cidades”. http://cidades.ibge.gov.br, consulté le 02/02/2017.

IBGE, 2017b. Base de données "produção agrícola e pecuária”. https://sidra.ibge.gov.br/home/couro/ brasil , consulté le 02/02/2017.

ICV, 2016. McDonald's anuncia compra de carne produzida em áreas com práticas sustentáveis, 17/08/2016. http://www.icv.org.br/2016/08/17/mcdonalds-anuncia-compra-de-carneproduzida-em-areas-com-praticas-sustentaveis/, consulté le 05/03/2017.

INPE, 2014, Projeto TerraClass. http://www.inpe.br/cra/projetos_pesquisas/terraclass2014.php, consulté le 23/02/2017.

INPE, 2016. Projeto PRODES - Monitoramento da floresta amazônica brasileira por satélite. http:// www.obt.inpe.br/prodes/index.php, consulté le 10/11/2016.

Jarvis A., Reuter H.I., Nelson A., Guevara E., 2008. Hole-filled SRTM for the globe Version 4, available from the CGIAR-CSI SRTM 90m Database. http://srtm.csi.cgiar.org.

Lapola D., Martinelli L., Peres C., Ometto J., Ferreira M., Nobre C., Aguiar A.P., Bustamante M., Cardoso M., Costa M., Joly C., Leite C., Moutinho P., Sampaio G., Strassburg B., Vieira I., 2014. Pervasive transition of the Brazilian land-use system. Nature Climate Change, 4, p. 27-35. DOI : 10.1038/nclimate2056

Le Tourneau, F-M., 2015. Le Brésil maîtrise-t-il (enfin) la déforestation en Amazonie ? Cybergeo : European Journal of Geography, Environnement, Nature, 753. DOI : 10.4000/cybergeo.27325 
Macedo M., DeFries R., Morton D., Stickler C., Galford G., Shimabukuro Y., 2012. Decoupling of deforestation and soy production in the southern Amazon during the late 2000s. PNAS, 109, 4, p. 1341-1346. DOI : 10.1073/pnas.1111374109

MDA, 2011. Estatísticas do meio rural 2010-2011. Departamento Intersindical de Estatística e Estudos Socioeconômicos (DIEESE), Núcleo de Estudos Agrários e Desenvolvimento Rural (NEAD), 4e ed., Ministério do Desenvolvimento Agrário São Paulo, 292 p. http://bibspi.planejamento.gov.br/ bitstream/handle/iditem/707/Estatisticas_Meio_Rural_2011.pdf?sequence=3\%5D

MMA, 2014. Plano Nacional de Recuperação da Vegetação Nativa (Planaveg). Ministério do Meio Ambiente, Brazil, 79 p. http://www.mma.gov.br/images/arquivo/80049/Planaveg/

PLANAVEG_20-11-14.pdf

Nepstad D., McGrath D., Stickler C., Alencar A., Azevedo A., Swette B., Bezerra T., DiGiano M., Shimada J., Seroa da Motta R., Armijo E., Castello L., Brando P., Hansen M.C., McGrath-Horn M., Carvalho O., Hess L., 2014. Slowing Amazon deforestation through public policy and interventions in beef and soy supply chains. Science, 344, p. 1118-1123. DOI :10.1126/science.1248525

Perrier F., 2014. Caractérisation et cartographie de l'éco-efficience des pâturages amazoniens à l'aide d'images MODIS. Mémoire de Master 1 de géographie, Université du Maine, Le Mans, 65 p.

Piketty M.G., Veiga J.B., Tourrand J.F., Alves A.M.N., Poccard-Chapuis R., Thâles M., 2005. Les déterminants de l'expansion de l'élevage bovin en Amazonie Orientale : conséquences pour les politiques publiques. Cahiers Agricultures, 14, 1, p. 90-95.

Piketty M.G., Poccard-Chapuis R., Drigo I., Coudel E., Plassin S., Laurent F., Thâles M., 2015. Multilevel Governance of Land Use Changes in the Brazilian Amazon: Lessons from Paragominas, State of Pará. Forests, 6, p. 1516-1536. DOI :10.3390/f6051516

Piketty M.G., Piraux M., Blanc L., Laurent F., Cialdella N., Ferreira J., Coudel E., Mazzei L.F, Resque G.A.L., Le Page C., Naudin K., Carvalho S.A., Gomes M., Poccard-Chapuis R., 2017. «Municípios Verdes » : passer du zéro-déforestation à la gestion durable des ressources naturelles en Amazonie brésilienne. In : Caron P., Valette E., Wassenar T., Coppens G., Papazian V., Des territoires vivants pour transformer le monde, Quae (sous presse).

Pinto A., Amaral P., Souza Jr. C., Verissimo A., Salomão R., Gomes G., Balieiro C., 2009. Diagnóstico socioeconômico e florestal de Paragominas. Instituto do Homem e Meio Ambiente da Amazônia Imazon, Belém/PA, 65 p.

Piraux M., Cialdella N., Poccard R., Assis W., 2015. o futuro incerto da AF na Amazônia Brasileira, um desafio para territórios e políticas públicas. In Agricultura Familiar: ruralidade, território $e$ política pública/IICA, FÓRUM DRS, IICA, 2015 - Série Desenvolvimento Rural Sustentável, Brasília, 23, p. 85-92.

Pires M. O., 2013. O cadastro ambiental rural: das origens às perspectivas para a política ambiental. Conservação Internacional, Brasília, 24 p. http://www.inovacar.org.br/wp-content/uploads/ 2016/11/Cadastro-Ambiental-Rural-Origens-e-Perspectivas.pdf

Pires G.F., Abrahão G.M., Brumatti L.M., Oliveira L.J.C., Costa M.H., Liddicoat S., Kato E., Ladle R.J., 2016. Increased climate risk in Brazilian double cropping agriculture systems: Implications for land use in Northern Brazil. Agricultural and Forest Meteorology, 228-299, p. 286-298. DOI : 10.1016/ j.agrformet.2016.07.005

Poccard-Chapuis R., Bendahan A., Carvalho S., Navegantes L., Thâles M., Ferreira L., Vaz V., Plassin S., Tourrand J.F., 2015. Amazonie, la forêt qui cache la prairie. Pâturages Tech. Cult., 63, p. 150-167. 
Richardson V.A., Peres C.A., 2016. Temporal Decay in Timber Species Composition and Value in Amazonian Logging Concessions. PLoS ONE, 11, 7, e0159035. DOI : 10.1371/journal.pone.0159035

Rudorff B.F.T., Adami M., Aguiar D.A., Moreira M.A., Mello M.P., Fabiani L., Amaral D.F., Pires B.M., 2011. The Soy Moratorium in the Amazon Biome Monitored by Remote Sensing Images. Remote Sensing, 3, p. 185-202. DOI : 10.3390/rs3010185

SEMAS (Secretaria de Estado de Meio Ambiente e Sustentabilidade), 2016. Base de dados de imóveis rurais (Cadastro Ambiental Rural do Pará - CAR). Governo do Pará, Consulté en 08/2016. http:// car.semas.pa.gov.br/\#/consulta/mapa

Serviço Florestal Brasileiro, 2017. Boletim Informativo, até 28 de Fevereiro de 2017. http:// www.florestal.gov.br/documentos/car/boletim-do-car/2603-boletim-informativo-car-fevereirode-2017/file

Sist P., Mazzei L., Sablayrolles P., 2013. Supporting farm forestry. Perspective, 22, 4 p.

Souza C. Jr., Siqueira J.V., Sales M.H., Fonseca A.V., Ribeiro J.G., Numata I., Cochrane M.A., Barber C.P., Roberts D.A., Barlow J., 2013. Ten-Year Landsat Classification of Deforestation and Forest Degradation in the Brazilian Amazon. Remote Sensing, 5, 11, p. 5493-5513.

Strassburg B.B.N., Latawiec A.E., Barioni L.G., Nobre C.A., da Silva V.P., Valentim J.F., Vianna M., Assad E.D., 2014. When enough should be enough: Improving the use of current agricultural lands could meet production demands and spare natural habitats in Brazil. Global Environmental Change, 28, p. 84-97. DOI : 10.1016/j.gloenvcha.2014.06.001

Théry H., 1997. Routes et déboisement en Amazonie brésilienne: Rondônia 1974-1996. Mappemonde, 97, 3, p. 35-40.

Tonneau J-P., Piraux M., Coudel E., Guilherme de Azevedo S., 2009. Évaluation du développement territorial comme processus d'innovation et d'institutionnalisation : le cas du Territoire du Alto Sertão do Piauí e Pernambuco au Nordeste du Brésil. Vertigo: la revue électronique en sciences de l'environnement, 9, 3. DOI : 10.4000/vertigo.9207

Tourrand J.F., Vaz V., Da Veiga J.B., De Carvalho S.A., Quanz D., Poccard-Chapuis R., 2013. Pionniers d'Amazonie : une vision particulière de l'incertitude. In Ancey V., Avelange I., Dedieu B., Agir en situation d'incertitude en agriculture : regards pluridisciplinaires au Nord et au Sud. Bruxelles, PIE-Peter Lang, p. 213-228.

Tritsch I., Arvor D., 2016. Transition in environmental governance in the Brazilian Amazon: emergence of a new pattern of socio-economic development and deforestation. Land Use Policy, 59, p. 446-455. DOI : 10.1016/j.landusepol.2016.09.018

Tritsch I., Sist P., Narvaes I. D. S., Mazzei L., Blanc L., Bourgoin C., Cornu G., Gond V., 2016. Multiple patterns of forest disturbance and logging shape forest landscapes in Paragominas, Brazil. Forests, 7, 315, 15 p. http://dx.doi.org/10.3390/f7120315

Verissimo A., Barreto P., Mattos M., Tarifa R., Uhl C., 1992. Logging Impacts and prospects for sustainable forest management in an old Amazonian frontier: the case of Paragominas. Forest Ecology and Management, 55, 1-4, p. 169-199. https://doi.org/10.1016/0378-1127(92)90099-U

Viana C., Coudel E., Barlow J., Ferreira J., Gardner T., Parry L., 2016. How does hybrid governance emerge? Role of the elite in building a Green Municipality in the Eastern Brazilian Amazon. Environmental Policy and Governance, 26, 5, p. 337-350. DOI : 10.1001/eet.1720

Watanabe P., 2017. Cadastro de propriedades rurais não impede desmate ilegal na Amazônia. Folha de São Paulo, 02/01/2017, http://www1.folha.uol.com.br/ambiente/2017/01/1846064cadastro-de-propriedades-rurais-nao-impede-desmate-ilegal-na-amazonia.shtml 


\section{NOTES}

1. Une municipalité (município) au Brésil, correspond à la plus petite division administrative du pays, après les États fédérés.

2. Ses limites ont été définies en 1953. L'Amazonie Légale est une aire géographique d'environ 5 millions de $\mathrm{km}^{2}$ et couvre $61 \%$ du territoire brésilien. Elle est définie en fonction de similarités socio-économiques et comprend tous les États de la région Nord (Acre, Amapá, Amazonas, Pará, Rondônia, Roraima) ainsi que deux États de la région Centre-Ouest (Mato Grosso et Tocantins) et une partie de l'État du Maranhão (de la région Nordeste).

3. Date ultérieurement alignée sur celle décrétée par le nouveau Code Forestier adopté en 2012, qui prend comme référence le déboisement réalisé jusqu'au 22 juillet 2008.

4. Le CAR, Cadastre Environnemental Rural, trouve son origine dans le Système de Licence Environnementale des Propriétés Rurales (SLAPR), outil de suivi et de contrôle de la législation environnementale qui constitue la première initiative de cadastre électronique géoréférencé des propriétés rurales au Brésil, mis en œuvre dans l'État du Mato Grosso dans les années 2000. Pionnier, le programme a entraîné de nombreuses adaptations dans les autres États amazoniens comme le Pará qui en 2006 créé son propre cadastre et qui pour la première fois utilise le terme de CAR (Pires, 2013). Le CAR actuel devient obligatoire avec l'art. 29 du nouveau Code Forestier (BRASIL, 2012) pour tous les propriétaires ruraux du pays. Il s'agit d'un registre électronique, dans lequel le propriétaire déclare les principales zones environnementales de sa propriété et fait état de sa conformité (ou non) aux réglementations. L'objectif du CAR est de fournir une sorte de radiographie de toutes les propriétés rurales du pays, favorisant ainsi le contrôle et le suivi de leur régularisation. Il permet en outre d'avoir une idée précise de la taille de la dette environnementale (passivo ambiental) des propriétaires ruraux du pays.

5. L'obligation de présenter une licence environnementale (LAR, Licenciamento ambiental rural) pour toutes les activités rurales agricoles a été mise en place en 2006 dans l'État du Pará. D'abord appelée licence d'activité rurale, elle est devenue une licence environnementale d'activité rurale. L'enregistrement dans les bases du CAR est une condition sine qua non à l'obtention de cette licence.

6. Il définit notamment les règles concernant les zonages environnementaux présents au sein de la propriété : la Réserve Légale (RL) et l'Aire de Protection Permanente (APP). Ces deux zonages ont été définis dans un premier temps dans le Code Forestier de 1965, puis ont subi des changements majeurs lors de la réforme du Code Forestier. La Réserve Légale, correspond pour l'Amazonie à $80 \%$ de la propriété (avec une exception à $50 \%$ pour les propriétés qui avaient déjà été déforestées avant le 22 juillet 2008), en théorie donc, la production ne concerne que $20 \%$ des terres. L'APP correspond aux franges ripariennes des cours d'eau et aux pentes raides, que les propriétaires ruraux doivent impérativement préserver. Sa largeur dépend d'une série de variables (largeur du cours d'eau, taille de la propriété, localisation de celle-ci et date des derniers déboisements).

7. Créé en 2008 par le gouvernement brésilien, le Fonds Amazonie est alimenté par des dons d'États étrangers, en premier lieu ceux de la Norvège qui s'élèvent à un milliard de dollars, ou ceux de grandes entreprises comme la Petrobras. Le fonds subventionne les actions de contrôle de la déforestation et des projets de gestion durable des forêts, de restauration des zones dégradées, de régularisation foncière et de développement rural, selon les priorités et critères choisis par le gouvernement brésilien.

8. Les propriétés cadastrées sont enregistrées dans un système appelé SiCAR, consultable en ligne: http://www.car.gov.br/publico/imoveis/index (consulté le 24/03/2017).

9. L'INCRA estime en 2009 qu'il y a 172 millions d'hectares de propriétés formelles (título) et informelles (posse) dans la région Nord (MDA, 2011). Les surfaces cadastrées (CAR) en 2017 représentent donc près de $2 / 3$ des surfaces privées. 
10. l'açaí, Euterpe oleracea, est un palmier produisant des fruits comestibles, bien valorisés depuis quelques années sur les marchés régionaux et internationaux.

11. Les chiffres de la déforestation globale sont calculés pour le biome de forêt amazonienne au Brésil (i.e. les cerrados ne sont pas pris en compte). Pour pouvoir les croiser avec les statistiques agricoles mesurées aux échelons administratifs (État, municipalité), nous avons choisi de ne travailler que sur la région Nord et le Mato Grosso où le biome forestier est dominant.

\section{ABSTRACTS}

The Amazon has recently experienced a dramatic decline in deforestation coupled with economic development. However, this process is uneven. While large and medium-sized producers are intensifying and diversifying their production systems in the already cleared areas, thus apparently abandoning expansion strategies at the moment, family farming remains aloof from this decoupling between production and deforestation. In this article, the commitments of public authorities and sectors, their effects on deforestation and inequalities of adaptation are analyzed at the scale of the Legal Amazon and in a municipality considered as a model because involved in a Município Verde initiative: Paragominas, in the Eastern Amazon.

L'Amazonie a amorcé depuis quelques années une baisse de la déforestation couplée à un développement économique. Le processus est cependant inégal. Si les grands et moyens producteurs font évoluer leurs systèmes de production vers l'intensification et la diversification dans les espaces déjà ouverts, délaissant en apparence les stratégies d'expansion, l'agriculture familiale reste à l'écart du découplage entre production et déforestation. Dans cet article, les engagements des pouvoirs publics et des filières, leurs effets en matière de déforestation et les inégalités d'adaptation sont analysés à l'échelle de l'Amazonie Légale et à celle d'une municipalité considérée comme un modèle car à l'initiative d'une démarche de Município Verde: Paragominas, en Amazonie orientale.

\section{INDEX}

Keywords: deforestation, agricultural frontier, agriculture, livestock, public policy, Amazon Mots-clés: déforestation, front pionnier, agriculture, élevage, politique publique, Amazonie

\section{AUTHORS}

\section{FRANÇOIS LAURENT}

François Laurent, Francois.Laurent@univ-lemans.fr, est Professeur à l'Université du Maine, UMR ESO. Il a publié récemment :

- Laurent, F., Poccard-Chapuis, R., Plassin, S., Pimentel Martinez, G., 2016. Soil texture derived from topography in North-eastern Amazonia. Journal of Maps, 13(2), p. 109-115. http:// www.tandfonline.com/doi/full/10.1080/17445647.2016.1266524

- Piketty M.G., Piraux M., Blanc L., Laurent F., Cialdella N., Ferreira J., Coudel E., Mazzei L.F., 
Resque G.L., Lepage C., Naudin K., Carvalho S.A., Gomes M., Poccard-Chapuis R., 2017.

« Municipios Verdes »: from zero deforestation to the sustainable management of natural resources in the Brazilian Amazon. In P. Caron, E. Valette, T. Wassenaar, G. C. d'Eeckenbrugge and V. Papazian coordinators, Living territoires to transform the world, Ed. QUAE, coll. Agricultures et défis du monde CIRAD/AFD, $272 \mathrm{p}$.

Laurent F., 2015. L'Agriculture de Conservation et sa diffusion en France et dans le monde. Cybergeo : European Journal of Geography [En ligne], Environnement, Nature, Paysage, document 747 , mis en ligne le 10 novembre 2015. http://cybergeo.revues.org/27284. DOI : 10.4000/ cybergeo.27284.

\section{DAMIEN ARVOR}

Damien Arvor, damien.arvor@gmail.com, est Chargé de Recherches CNRS, Université Rennes 2, UMR LETG. Il a publié récemment :

- Arvor D., Funatsu, B., Michot V., Dubreuil. V. 2017. Monitoring Rainfall Patterns in the Southern Amazon with PERSIANN-CDR Data: Long-Term Characteristics and Trends. Remote Sensing, 9, 889. - Arvor D., Tritsch I., Barcellos C., Jégou N., Dubreuil V. 2017. Land use sustainability on the South-Eastern Amazon agricultural frontier : Recent progress and the challenges ahead. Applied Geography, 80, p. 86-97.

- Arvor D., Daugeard M., Tritsch I., de Mello-Théry N.A., Théry H., Dubreuil V. 2016. Combining socioeconomic development with environmental governance in the Brazilian Amazon : the Mato Grosso agricultural frontier at a tipping point. Environment, Development and Sustainability. p. 1-22.

\section{MARION DAUGEARD}

Marion Daugeard est doctorante au Centre de Recherche et de Documentation sur les Amériques (CREDA-UMR 7227, Université Paris 3 Sorbonne Nouvelle) en cotutelle avec Centro de Desenvolvimento Sustentável (CDS, Universidade de Brasília). Elle a publié récemment : - Arvor D., Daugeard M., Tritsch I. et al., 2016. Combining socioeconomic development with environmental governance in the Brazilian Amazon: the Mato Grosso agricultural frontier at a tipping point. Environment, Development and Sustainability, p. 1-22. DOI: 10.1007/s10668-016-9889-1.

\section{REINIS OSIS}

Reinis Osis est doctorant à l'Université du Maine, UMR ESO. Il a publié récemment :

- Osis, R., Laurent, F., Poccard-Chapuis R., 2017. Spatial relationships between natural resources and land use dynamics in the amazonian agricultural frontier. 9th International Workshop on the Analysis of Multitemporal Remote Sensing Images (MultiTemp), Bruges, Belgique. DOI: 10.1109/MultiTemp.2017.8035242

- Lima, R., Osis R., Queiroz A.R., Santos A.H.M., 2016. Least-cost path Analysis and Multi-criteria Assessment for Routing Electricity Transmission Lines. IET Generation Transmission \& Distribution, 10(16). DOI: 10.1049/iet-gtd.2016.1119

- Osis R., Lima R., Dzejdzej M., Santos A.H.M., ; Czank Junior, L.; Carvalho Junior, E.D. Rodrigues, L.S. , 2016. Mapeamento de valores de Nspt como subsídio ao planejamento de linhas de transmissão na Amazônia Oriental. Geotecnia, n 137.

\section{ISABELLE TRITSCH}

Isabelle Tritsch est post-doc au CIRAD, UR Forêts et Sociétés, Montpellier. Elle a publié récemment :

- Tritsch I., Sist P., Narvaes I., Mazzei L., Blanc L., Bourgoin C., Cornu G., Gond V., 2016. Multiple patterns of forest disturbance and logging are shaping forest landscapes. Forests, 7(12), p. 315. 
- Tritsch I., Arvor D., 2016. Transition in environmental governance in the Brazilian Amazon: emergence of a new pattern of socio-economic development and deforestation. Land Use Policy, 59, p. 446-455.

- Tritsch I., Le Tourneau F-M., 2016. Population densities and deforestation in the Brazilian Amazon: new insights on the current human settlement patterns. Applied Geography, 76, p. 163-172.

\section{EMILIE COUDEL}

Emilie Coudel est chercheure au Cirad. Elle a publié récemment :

- Coudel E., Tonneau J.P., Bousquet F., Caniello M., Chia E., Daré W., Gurung T.R., Jankowski F., Leal F., Le Page C., Piraux M., Rey-Valette H., Sabourin E., Trébuil G. 2017. Social Learning for Territorial Development. In Caron P., Valette E, Wassenaar T., Coppens G., Papazian V. (eds.). Living territories to transform the world. Versailles, Ed. Quae, p. 161-166. (Agricultures et défis du monde).

- Viana C., Coudel E., Barlow J., Ferreira J., Gardner T., Parry L. 2016. How does hybrid governance emerge? Role of the elite in building a green municipality in the Eastern Brazilian Amazon. Environmental Policy and Governance, 26 (5), p. 337-350.

- Coudel E., Amazonas M., Ferreira J., Eloy L., Hercowitz M., Mattos L., May P., Muradian R., Piketty M.G., Toni F. 2017. The rise of PES in Brazil: from pilot projects to public policies. In Ezzine de Blas D., Le Coq JF., Guevara A (Coords.), Los pagos por servicios ambientales en américa latina: gobernanza, impactos y perspectivas. Mexico, Universidad Iberoamericana, p. 129-164.

\section{MARIE-GABRIELLE PIKETTY}

Marie-Gabrielle Piketty, marie-gabrielle.piketty@cirad.fr, est économiste au Cirad. Elle a publié récemment :

- Morello T.F., Piketty M.G., Gardner T., Parry L., Barlow J., Ferreira J., Tancredi N.S., 2018. Fertilizer Adoption by Smallholders in the Brazilian Amazon: Farm-level Evidence. Ecological Economics, n 144 , p. 278-291.

- Piketty M.G., Piraux M., Blanc L., Laurent F., Cialdella N., Ferreira J., Coudel E., Mazzei L.F., Resque G.L., Lepage C., Naudin K., Carvalho S.A., Gomes M., Poccard-Chapuis R., 2017.

« Municipios Verdes »: from zero deforestation to the sustainable management of natural resources in the Brazilian Amazon. In P. Caron, E. Valette, T. Wassenaar, G. C. d'Eeckenbrugge and V. Papazian coordinators, Living territoires to transform the world. Ed. QUAE, coll. Agricultures et défis du monde CIRAD/AFD, $272 \mathrm{p}$.

- Piketty M.G., Poccard-Chapuis R., Drigo I., Coudel E., Plassin S., Laurent F., Thâles M., 2015. Multi-level governance of land use changes in the Brazilian Amazon: Lessons from Paragominas, State of Pará. Forests, 6 (5), p. 1516-1536.

\section{MARC PIRAUX}

Marc Piraux est chercheur à l'UMR Tetis - CIRAD et Professeur à l'UFPA, Belem, Brésil. Il a publié récemment :

- Piraux M., Tonneau J.P., Sabourin E., Chia E., Caniello M., Polge E., Massardier G. 2017. Les enjeux de la gouvernance territoriale : l'exemple du Brésil rural. In Caron Patrick (ed.), Valette Elodie (ed.), Wassenaar Tom (ed.), Coppens D'Eeckenbrugge Géo (ed.), Papazian Vatché (ed.), Des territoires vivants pour transformer le monde. Versailles, Ed. Quae, p. 187-190.

- Polge E, Piraux M., 2017. Analyse des dynamiques d'interaction dans les dispositifs de gouvernance territoriale en Amazonie Brésilienne, Quels enseignements pour le programme Territoires de la citoyenneté ? Canadina journal of regional science. 
- Blanc L., Ferreira J., Piketty M.G., Bourgoin C., Gond V., Hérault B., Kanashiro M., Laurent F., Piraux M., Rutishauser E., Sist P. 2017. Managing degraded forests, a new priority in the Brazilian Amazon. Perspective - Cirad (40), 4 p. http://dx.doi.org/10.18167/agritrop/00042.

\section{CECILIA VIANA}

Centro de Desenvolvimento Sustentável (CDS), Campus Darcy Ribeiro, Universidade de Brasília, Brésil.

\section{VINCENT DUBREUIL}

Vincent Dubreuil, vincent.dubreuil@univ-rennes2.fr, est Professeur à l'Université Rennes 2, UMR LETG. Il a publié récemment :

- Dubreuil V., Funatsu B., Michot V., Nasuti S., Debortoli N., de Mello-Thery N.A., Le Tourneau F.M., 2017. Local rainfall trends and their perception by the Amazonian communities. Climatic Change, 143(3), p. 461-472.

- Arvor D., Daugeard M., Tritsch I., De Mello N.A., Thery H., Dubreuil V., 2016. Combining socioeconomic development with environmental governance in the Brazilian Amazon: the Mato Grosso agricultural frontier at a tipping point. Environ Dev Sustain, $22 \mathrm{p}$.

- Dubreuil V., De Mello-Thery N., 2014. Environnement et Géomatique : approches comparées FranceBrésil. Actes du colloque (dir.). Nov. 2014, Rennes-2, France. 502 p. http:// envibras2014.sciencesconf.org/conference/envibras2014/eda_fr.pdf

\section{ALI F. HASAN}

Ali F. Hassan est doctorant à l'Université du Maine, UMR ESO. Il a publié récemment : - Hasan, A.F., Laurent, F., Blanc, L., Messner F., 2017. The use of Landsat time series for identification of forest degradation levels in the eastern Brazilian Amazon (Paragominas). 9th International Workshop on the Analysis of Multitemporal Remote Sensing Images (MultiTemp), Bruges, Belgique. DOI: 10.1109/Multi-Temp.2017.8035243

\section{FRANÇOIS MESSNER}

François Messner est technicien à l'Université du Maine, UMR ESO. Il a publié récemment :

- Corbonnois J., Laurent F., Andreu-Boussut V., Messner F., Verdum R., Medeiros Vieira R., Sogue M., 2014. L'intensification des pratiques agricoles et la mobilisation des ressources naturelles dans la Pampa du sud du Brésil. [VertiGo] La revue électronique en sciences de l'environnement, vol. 14, $\mathrm{n}^{\circ} 1$.

- Alhaskeer Z., Corbonnois J., Messner F., LAURENT F., 2014. Elaboration d'une typologie des formes de vallées. Application au bassin de la Maine (Plateaux du Nord Ouest de la France). Revue Physio-Géo.

- Corbonnois J., Verdum R., Messner F., Laurent F., Garcez Soares V., 2011. L'érosion des sols sableux dans les campos du sud du Brésil (bassin de l'Ibicui, Rio Grande do Sul). Revue Géomorphologies. 